Acta Crystallographica Section E

Structure Reports

Online

ISSN 1600-5368

\section{Isogentisin (1,3-dihydroxy-7-methoxyxanthone)}

\author{
Ivana Radosavljević Evans, ${ }^{\mathrm{a} *}$ \\ Judith A. K. Howard, ${ }^{\text {a }}$ \\ Katarina Šavikin-Fodulović ${ }^{b}$ and \\ Nebojša Menkovićb

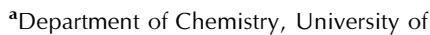 \\ Durham, South Road, Durham DH1 3LE, \\ England, and ${ }^{\mathbf{b}}$ Institute for Medicinal Plant \\ Research, Tadeuša Košćuška 1, 11000 Belgrade, \\ Serbia and Montenegro \\ Correspondence e-mail: \\ ivana.radosavljevic@durham.ac.uk
}

\author{
Key indicators \\ Single-crystal X-ray study \\ $T=120 \mathrm{~K}$ \\ Mean $\sigma(\mathrm{C}-\mathrm{C})=0.005 \AA$ \\ $R$ factor $=0.034$ \\ $w R$ factor $=0.117$ \\ Data-to-parameter ratio $=10.8$
}

For details of how these key indicators were automatically derived from the article, see http://journals.iucr.org/e.
(C) 2004 International Union of Crystallography Printed in Great Britain - all rights reserved
The crystal structure of isogentisin, $\mathrm{C}_{14} \mathrm{H}_{10} \mathrm{O}_{5}$, a natural product isolated from Gentiana lutea, has been determined. The phenolic ring system is essentially planar and the displacement of the methoxy substituent from the mean molecular plane is very small. The structure is stabilized by a one-dimensional chain of intermolecular hydrogen bonds.

\section{Comment}

Xanthone compounds commonly occur in several higher plant families, such as Gentianaceae, Guttiferae, Moraceae and Polygalaceae. The study of xanthones is interesting both from the chemosystematic and pharmacological point of view. Inhibition of Type A and Type B monoamine oxidases (MAO) by a number of xanthones has been observed (Suzuki et al., 1980, 1981). Among the xanthones that have been tested, isogentisin revealed potent MAO inhibition (Suzuki et al., 1978). Four ethanolic extracts prepared from leaves, flowers and roots of Gentiana lutea were tested for antitubercular activity against Mycobacterium bovis (BCG-strain). The extract obtained from flowers showed strong inhibition at a concentration of $1000 \mu \mathrm{g} \mathrm{ml}^{-1}$ and slight inhibition at $500 \mu \mathrm{g} \mathrm{ml}^{-1}$. This activity increased during the various purification steps, which finally led to the isolation of the active compound isogentisin (Menković et al., 1999). Mutagenicity in the Ames test in Salmonella typhimurium was also shown for isogentisin (Morimoto et al., 1983, Matsushima et al., 1985). Isogentisin was first isolated by Cannonica \& Pelizzoni (1955). The present paper presents the first single-crystal X-ray analysis of isogentisin and confirms that the crystal structure correpsonds to 1,3-dihydroxy-7-methoxyxanthone, (I) (Fig. 1). The 1,3-dihydroxy-7-methoxyxanthone fragment is essentially planar, with the largest displacement within the phenolic ring system of 0.062 (3) $\AA$ for $\mathrm{C} 1$. The methyl group of the methoxy substituent lies close to the mean plane of the molecule, as shown by the torsion angle of $\mathrm{C} 10-\mathrm{C} 9-\mathrm{O} 15-\mathrm{C} 19$ of $5.2(5)^{\circ}$.<smiles>COc1ccc2oc3cc(O)cc(O)c3c(=O)c2c1</smiles>

(I)

The packing diagram for isogentisin is shown in Figs. 2 and 3. The crystal structure can be described in terms of parallel molecules stacked along the direction of the $a$ crystallographic axis, with the normal to the plane forming an angle of about $20^{\circ}$ relative to it, and an intermolecular separation of about $3.5 \AA$. Within a xanthone unit, an intramolecular hydrogen bond with a length of $1.91 \AA$ exists between the hydroxyl $\mathrm{H}$
Received 3 August 2004 Accepted 10 August 2004 Online 21 August 2004 
Figure 1

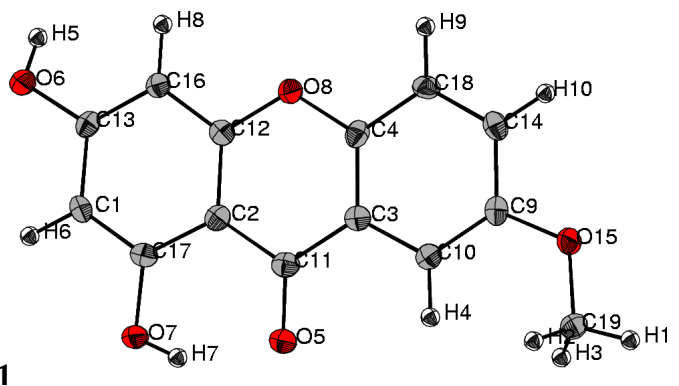

The molecular structure of isogentisin and the atom-numbering scheme. Displacement ellipsoids are drawn at the $50 \%$ probability level.

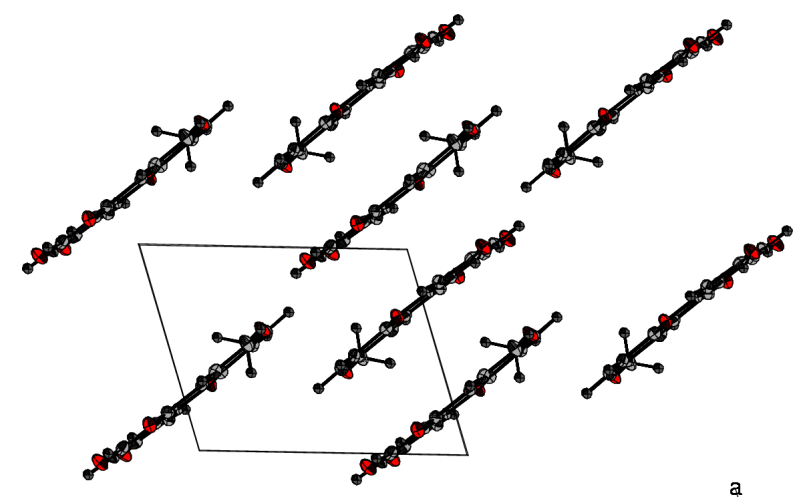

Figure 2

$\stackrel{a}{b} c$

One view of the packing diagram for isogentisin.

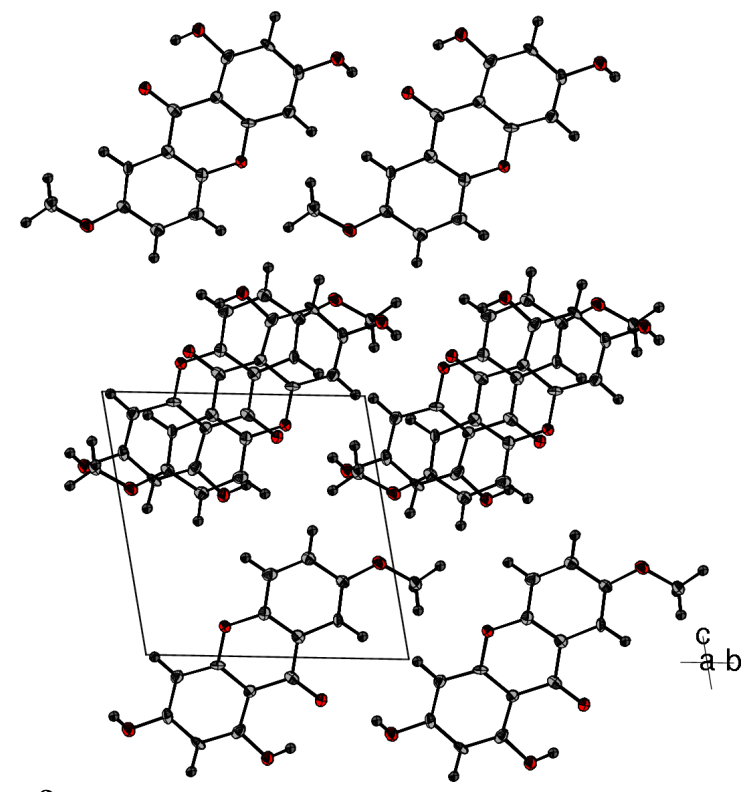

Figure 3

A second view of the packing diagram for isogentisin.

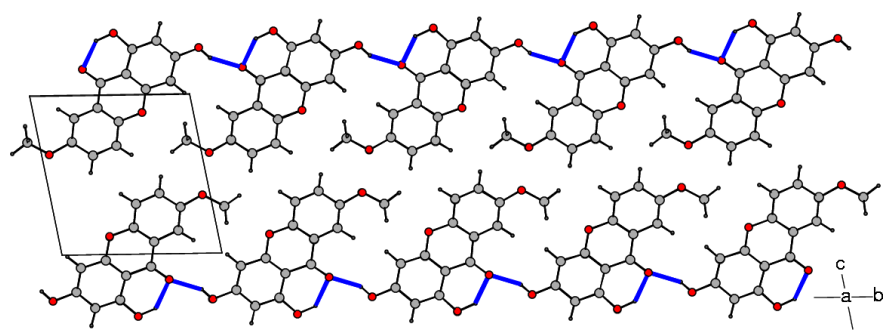

atom $\mathrm{H} 7$ and the $\mathrm{O} 5$ acceptor of an adjacent carbonyl group. In addition, the same carbonyl $\mathrm{O}$ atom participates in a onedimensional intermolecular hydrogen bond with the hydroxyl group on a neighbouring molecule (O5-H5 = $1.997 \AA$ ). The hydrogen-bonding patterns are shown in Fig. 4.

\section{Experimental}

Isolation of isogentisin from Gentiana lutea was carried out following a procedure described previously (Menković, 1997; Menković et al., 1990).

\section{Crystal data}

$\mathrm{C}_{14} \mathrm{H}_{10} \mathrm{O}_{5}$

$M_{r}=258.23$

Triclinic, $P \overline{1}$

$a=7.2287$ (14) $\AA$

$b=8.6286(15) \mathrm{A}$

$c=9.0370(16) \AA$

$\alpha=97.896(5)^{\circ}$

$\beta=105.962(6)^{\circ}$

$\gamma=97.698(5)^{\circ}$

$V=528.00(17) \AA^{3}$

$Z=2$

$D_{x}=1.611 \mathrm{Mg} \mathrm{m}^{-3}$

Mo $K \alpha$ radiation

Cell parameters from 826

reflections

$\theta=6.0-49.1^{\circ}$

$\mu=0.12 \mathrm{~mm}^{-1}$

$T=120 \mathrm{~K}$

Needle, yellow

$0.08 \times 0.04 \times 0.02 \mathrm{~mm}$

\section{Data collection}

Bruker SMART 6000

diffractometer

$\omega$ scans

Absorption correction: multi-scan (SADABS; Sheldrick, 1996)

$T_{\min }=0.956, T_{\max }=1.000$

4830 measured reflections

\section{Refinement}

Refinement on $F^{2}$

$R\left[F^{2}>2 \sigma\left(F^{2}\right)\right]=0.034$

$w R\left(F^{2}\right)=0.117$

$S=0.96$

1858 reflections

172 parameters

1866 independent reflections 783 reflections with $I>2 \sigma(I)$

$R_{\text {int }}=0.01$

$\theta_{\max }=25.0^{\circ}$

$h=-8 \rightarrow 8$

$k=-10 \rightarrow 10$

$l=-10 \rightarrow 10$

$\mathrm{H}$-atom parameters constrained Weighting scheme: see text $(\Delta / \sigma)_{\max }=0.001$

$\Delta \rho_{\max }=0.69 \mathrm{e} \AA^{-3}$

$\Delta \rho_{\min }=-0.59 \mathrm{e} \AA^{-3}$

Table 1

Selected geometric parameters $\left(\AA{ }^{\circ}\right)$.

\begin{tabular}{|c|c|c|c|}
\hline $\mathrm{C} 1-\mathrm{C} 13$ & $1.392(4)$ & $\mathrm{O} 6-\mathrm{C} 13$ & $1.352(4)$ \\
\hline $\mathrm{C} 1-\mathrm{C} 17$ & $1.369(4)$ & $\mathrm{O} 7-\mathrm{C} 17$ & $1.349(4)$ \\
\hline $\mathrm{C} 2-\mathrm{C} 11$ & $1.441(4)$ & $\mathrm{O} 8-\mathrm{C} 12$ & $1.364(3)$ \\
\hline $\mathrm{C} 2-\mathrm{C} 12$ & $1.401(4)$ & $\mathrm{C} 9-\mathrm{C} 10$ & $1.376(4)$ \\
\hline $\mathrm{C} 2-\mathrm{C} 17$ & $1.425(4)$ & $\mathrm{C} 9-\mathrm{C} 14$ & $1.400(5)$ \\
\hline $\mathrm{C} 3-\mathrm{C} 4$ & $1.387(4)$ & $\mathrm{C} 9-\mathrm{O} 15$ & $1.362(4)$ \\
\hline $\mathrm{C} 3-\mathrm{C} 10$ & $1.404(4)$ & $\mathrm{C} 12-\mathrm{C} 16$ & $1.376(4)$ \\
\hline $\mathrm{C} 3-\mathrm{C} 11$ & $1.454(4)$ & $\mathrm{C} 13-\mathrm{C} 16$ & $1.390(4)$ \\
\hline $\mathrm{C} 4-\mathrm{O} 8$ & $1.373(4)$ & $\mathrm{C} 14-\mathrm{C} 18$ & $1.372(4)$ \\
\hline $\mathrm{C} 4-\mathrm{C} 18$ & $1.401(4)$ & $\mathrm{O} 15-\mathrm{C} 19$ & $1.422(4)$ \\
\hline $\mathrm{O} 5-\mathrm{C} 11$ & $1.256(4)$ & & \\
\hline $\mathrm{C} 13-\mathrm{C} 1-\mathrm{C} 17$ & $119.9(3)$ & $\mathrm{C} 3-\mathrm{C} 11-\mathrm{O} 5$ & $122.0(3)$ \\
\hline $\mathrm{C} 11-\mathrm{C} 2-\mathrm{C} 12$ & $120.8(3)$ & $\mathrm{C} 2-\mathrm{C} 11-\mathrm{O} 5$ & $122.2(3)$ \\
\hline $\mathrm{C} 11-\mathrm{C} 2-\mathrm{C} 17$ & $122.4(3)$ & $\mathrm{C} 2-\mathrm{C} 12-\mathrm{O} 8$ & $121.5(3)$ \\
\hline $\mathrm{C} 12-\mathrm{C} 2-\mathrm{C} 17$ & $116.8(3)$ & $\mathrm{C} 2-\mathrm{C} 12-\mathrm{C} 16$ & $122.8(3)$ \\
\hline $\mathrm{C} 4-\mathrm{C} 3-\mathrm{C} 10$ & $118.7(3)$ & $\mathrm{O} 8-\mathrm{C} 12-\mathrm{C} 16$ & $115.7(3)$ \\
\hline $\mathrm{C} 4-\mathrm{C} 3-\mathrm{C} 11$ & $119.7(3)$ & $\mathrm{C} 1-\mathrm{C} 13-\mathrm{O} 6$ & $116.8(3)$ \\
\hline $\mathrm{C} 10-\mathrm{C} 3-\mathrm{C} 11$ & $121.6(3)$ & $\mathrm{C} 1-\mathrm{C} 13-\mathrm{C} 16$ & $121.1(3)$ \\
\hline $\mathrm{C} 3-\mathrm{C} 4-\mathrm{O} 8$ & $122.8(3)$ & $\mathrm{O} 6-\mathrm{C} 13-\mathrm{C} 16$ & $122.0(3)$ \\
\hline $\mathrm{C} 3-\mathrm{C} 4-\mathrm{C} 18$ & $121.4(3)$ & $\mathrm{C} 9-\mathrm{C} 14-\mathrm{C} 18$ & $120.2(3)$ \\
\hline $\mathrm{O} 8-\mathrm{C} 4-\mathrm{C} 18$ & $115.8(3)$ & C9-O15-C19 & $117.9(2)$ \\
\hline $\mathrm{C} 4-\mathrm{O} 8-\mathrm{C} 12$ & $119.4(2)$ & $\mathrm{C} 13-\mathrm{C} 16-\mathrm{C} 12$ & $118.4(3)$ \\
\hline $\mathrm{C} 10-\mathrm{C} 9-\mathrm{C} 14$ & $120.6(3)$ & $\mathrm{C} 2-\mathrm{C} 17-\mathrm{C} 1$ & $121.0(3)$ \\
\hline $\mathrm{C} 10-\mathrm{C} 9-\mathrm{O} 15$ & $125.2(3)$ & $\mathrm{C} 2-\mathrm{C} 17-\mathrm{O} 7$ & $120.8(3)$ \\
\hline $\mathrm{C} 14-\mathrm{C} 9-\mathrm{O} 15$ & $114.2(3)$ & $\mathrm{C} 1-\mathrm{C} 17-\mathrm{O} 7$ & $118.2(3)$ \\
\hline $\mathrm{C} 3-\mathrm{C} 10-\mathrm{C} 9$ & $120.0(3)$ & $\mathrm{C} 4-\mathrm{C} 18-\mathrm{C} 14$ & $119.1(3)$ \\
\hline $\mathrm{C} 3-\mathrm{C} 11-\mathrm{C} 2$ & $115.8(3)$ & & \\
\hline
\end{tabular}

Figure 4

Hydrogen bonding in isogentisin. 
Table 2

Hydrogen-bonding geometry $\left(\AA{ }^{\circ}\right)$.

\begin{tabular}{lllll}
\hline$D-\mathrm{H} \cdots A$ & $D-\mathrm{H}$ & $\mathrm{H} \cdots A$ & $D \cdots A$ & $D-\mathrm{H} \cdots A$ \\
\hline O6-H5 $\cdots$ O5 $^{\mathrm{i}}$ & 0.82 & 2.00 & $2.738(3)$ & 150 \\
$\mathrm{O} 7-\mathrm{H} 7 \cdots \mathrm{O} 5$ & 0.82 & 1.91 & $2.634(3)$ & 147 \\
\hline
\end{tabular}

Symmetry code: (i) $x, y-1, z$.

A Chebychev polynomial (Carruthers \& Watkin, 1979; Prince, 1982) was used for the weighting scheme, with $w=1.0 /\left[A_{0} T_{0}(x)+\right.$ $\left.\left.A_{1} \mathrm{~T}_{1}(x) \ldots+A_{n-1}\right] T_{n-1}(x)\right]$ where $A_{i}$ are the Chebychev coefficients listed below and $x=F_{\text {calc }} / F_{\max }$; robust weighting (Prince, 1982): $W=w\left[1-(\delta F / 6 \sigma F)^{2}\right]^{2}, A_{i}$ are $1.96,2.45$ and $0.676 . \mathrm{H}$ atoms were positioned geometrically $(\mathrm{C}-\mathrm{H}=1.0 \AA$ and $\mathrm{O}-\mathrm{H}=0.82 \AA)$ and refined using a riding model, with $U_{\text {iso }}(\mathrm{H})=1.2 U_{\text {eq }}(\mathrm{C})$ and $1.1 U_{\text {eq }}(\mathrm{O})$.

Data collection: SMART (Bruker, 1999); cell refinement: SAINT (Bruker, 1999); data reduction: SAINT (Bruker, 1999); program(s) used to solve structure: SIR92 (Altomare et al., 1994); program(s) used to refine structure: CRYSTALS (Betteridge et al., 2003); molecular graphics: ATOMS (Shape Software, 2000); software used to prepare material for publication: CRYSTALS.

\section{References}

Altomare, A., Cascarano, G., Giacovazzo, C., Guagliardi, A., Burla, M. C., Polidori, G. \& Camalli, M. (1994). J. Appl. Cryst. 27, 435.

Betteridge, P. W., Carruthers, J. R., Cooper, R. I., Prout, K. \& Watkin, D. J. (2003). J. Appl. Cryst. 36, 1487.

Bruker (1999). SMART (Version 5.049) and SAINT (Version 5.00). Bruker AXS Inc., Madison, Wisconsin, USA.

Cannonica, L. \& Pelizzoni, F. (1955). Gazzetta, 85, 1007-1024.

Carruthers, J. R. \& Watkin, D. J. (1979). Acta Cryst. A35, 698-699.

Matsushima, T., Araki, A., Yagame, O., Muramatsu, M., Koyama, K., Ohsawa, K., Natori, S. \& Tomimori, H. (1985). Mutation Res. 150, 141-146.

Menković, N. (1997). PhD thesis, University of Belgrade, Serbia and Montenegro.

Menković, N., Savikin-Fodulović, K. \& Cebedzić, R. (1999). Pharm. Pharmacol. Lett. 9, 74-75.

Morimoto, I., Nozaka, T., Watanabe, F., Ishino, M., Hirose, Y. \& Okitsu, T. (1983). Mutation Res. 116, 103-117.

Prince, E. (1982). Mathematical Techniques in Crystallography and Materials Science. New York: Springer-Verlag.

Shape Software (2000). ATOMS. Version 5.1. Shape Software, Kingsport, TN, USA.

Sheldrick, G. M. (1996). SADABS. University of Göttingen, Germany.

Suzuki, O., Katsumata, Y., Oya, M., Chari, V. M., Klapfenberger, R., Wagner, H. \& Hostettmann, K. (1978). Biochem. Pharm. 27, 2075-2078.

Suzuki, O., Katsumata, Y., Oya, M., Chari, V. M., Klapfenberger, R., Wagner, H. \& Hostettmann, K. (1980). Planta Med. 39, 19-23.

Suzuki, O., Katsumata, Y., Oya, M., Chari, V. M., Vermes, B., Wagner, H. \& Hostettmann, K. (1981). Planta Med. 42, 17-21. 


\section{supporting information}

Acta Cryst. (2004). E60, o1557-o1559 [https://doi.org/10.1107/S1600536804019890]

Isogentisin (1,3-dihydroxy-7-methoxyxanthone)

Ivana Radosavljević Evans, Judith A. K. Howard, Katarina Šavikin-Fodulović and Nebojša

Menković

1,3-dihydroxy-7-methoxyxanthone

Crystal data

$\mathrm{C}_{14} \mathrm{H}_{10} \mathrm{O}_{5}$

$Z=2$

$M_{r}=258.23$

Triclinic, $P \overline{1}$

Hall symbol: -P 1

$a=7.2287(14) \AA$

$b=8.6286(15) \AA$

$c=9.0370(16) \AA$

$\alpha=97.896(5)^{\circ}$

$\beta=105.962(6)^{\circ}$

$\gamma=97.698(5)^{\circ}$

$V=528.00(17) \AA^{3}$

$F(000)=268$

$D_{\mathrm{x}}=1.611 \mathrm{Mg} \mathrm{m}^{-3}$

Mo $K \alpha$ radiation, $\lambda=0.71073 \AA$

Cell parameters from 826 reflections

$\theta=6.0-49.1^{\circ}$

$\mu=0.12 \mathrm{~mm}^{-1}$

$T=120 \mathrm{~K}$

Needle, yellow

$0.08 \times 0.04 \times 0.02 \mathrm{~mm}$

Data collection

Bruker SMART 6000

diffractometer

Graphite monochromator

$\omega$ scans

Absorption correction: multi-scan

(SADABS; Sheldrick, 1996)

$T_{\min }=0.956, T_{\max }=1.000$

4830 measured reflections

1866 independent reflections

783 reflections with $I>2 \sigma(I)$

$R_{\text {int }}=0.01$

$\theta_{\max }=25.0^{\circ}, \theta_{\min }=2.4^{\circ}$

$h=-8 \rightarrow 8$

$k=-10 \rightarrow 10$

$l=-10 \rightarrow 10$

\section{Refinement}

Refinement on $F^{2}$

Least-squares matrix: full

$R\left[F^{2}>2 \sigma\left(F^{2}\right)\right]=0.034$

$w R\left(F^{2}\right)=0.117$

$S=0.96$

1858 reflections

172 parameters

0 restraints

Primary atom site location: structure-invariant direct methods

Hydrogen site location: inferred from neighbouring sites

H-atom parameters not refined

Chebychev polynomial (Carruthers, 1979;

Prince, 1982) $w=1.0 /\left[\mathrm{A}_{0} \mathrm{~T}_{0}(\mathrm{x})+\mathrm{A}_{1} \mathrm{~T}_{1}(\mathrm{x}) \cdots+\right.$ $\left.\left.\mathrm{A}_{\mathrm{n}-1}\right] \mathrm{T}_{\mathrm{n}-1}(\mathrm{x})\right]$

where $A_{i}$ are the Chebychev coefficients listed below and $\mathrm{x}=F / F \max$; robust weighting (Prince, 1982): $\mathrm{W}=w\left[1-(\delta F / 6 \sigma F)^{2}\right]^{2}, \mathrm{~A}_{\mathrm{i}}$ are $1.96,2.45$ and 0.676

$(\Delta / \sigma)_{\max }=0.001$

$\Delta \rho_{\max }=0.69 \mathrm{e} \AA^{-3}$

$\Delta \rho_{\min }=-0.59$ e $\AA^{-3}$ 
Fractional atomic coordinates and isotropic or equivalent isotropic displacement parameters $\left(\AA^{2}\right)$

\begin{tabular}{|c|c|c|c|c|}
\hline & $x$ & $y$ & $z$ & $U_{\text {iso }} * / U_{\text {eq }}$ \\
\hline $\mathrm{C} 1$ & $0.9594(4)$ & $0.1431(4)$ & 0.6659 (4) & 0.0173 \\
\hline $\mathrm{C} 2$ & $1.1318(5)$ & $0.3728(4)$ & $0.8682(4)$ & 0.0177 \\
\hline C3 & $1.3211(4)$ & $0.5934(3)$ & 1.0799 (3) & 0.0154 \\
\hline $\mathrm{C} 4$ & $1.3865(5)$ & 0.4805 & $1.1671(3)$ & 0.0181 \\
\hline $\mathrm{O} 5$ & $1.1236(3)$ & 0.6399 & $0.8389(2)$ & 0.0220 \\
\hline O6 & $0.9766(3)$ & -0.1150 & $0.7128(2)$ & 0.0230 \\
\hline $\mathrm{O} 7$ & $0.9354(3)$ & 0.3977 (3) & $0.6125(3)$ & 0.0235 \\
\hline O8 & $1.3262(3)$ & $0.3199(2)$ & $1.1131(2)$ & 0.0195 \\
\hline $\mathrm{C} 9$ & $1.5219(5)$ & $0.7988(4)$ & $1.2928(4)$ & 0.0179 \\
\hline $\mathrm{C} 10$ & $1.3905(5)$ & 0.7550 & $1.1453(4)$ & 0.0179 \\
\hline $\mathrm{C} 11$ & $1.1864(5)$ & $0.5423(4)$ & $0.9226(4)$ & 0.0184 \\
\hline $\mathrm{C} 12$ & $1.2023(4)$ & $0.2669(4)$ & $0.9653(3)$ & 0.0161 \\
\hline $\mathrm{C} 13$ & $1.0311(5)$ & $0.0431(4)$ & $0.7678(4)$ & 0.0178 \\
\hline $\mathrm{C} 14$ & $1.5887(5)$ & $0.6835(4)$ & $1.3786(4)$ & 0.0192 \\
\hline $\mathrm{O} 15$ & $1.6008(3)$ & $0.9514(2)$ & $1.3672(2)$ & 0.0216 \\
\hline $\mathrm{C} 16$ & $1.1517(4)$ & $0.1043(4)$ & $0.9191(4)$ & 0.0161 \\
\hline $\mathrm{C} 17$ & $1.0072(4)$ & $0.3046(4)$ & $0.7146(4)$ & 0.0168 \\
\hline C18 & $1.5215(5)$ & $0.5249(4)$ & $1.3170(3)$ & 0.0188 \\
\hline C19 & $1.5317(5)$ & $1.0763(4)$ & $1.2928(4)$ & 0.0231 \\
\hline H5 & $1.0557(3)$ & $-0.1619(2)$ & $0.7637(2)$ & $0.0250 *$ \\
\hline $\mathrm{H7}$ & $0.9795(3)$ & $0.4911(3)$ & $0.6531(3)$ & $0.0250 *$ \\
\hline H1 & $1.6017(5)$ & $1.1812(4)$ & 1.3599 (4) & $0.0265^{*}$ \\
\hline H3 & $1.5570(5)$ & $1.0684(4)$ & $1.1889(4)$ & $0.0265^{*}$ \\
\hline $\mathrm{H} 2$ & $1.3881(5)$ & $1.0671(4)$ & $1.2774(4)$ & $0.0265^{*}$ \\
\hline $\mathrm{H} 4$ & $1.3444(5)$ & $0.8384(4)$ & $1.0846(4)$ & $0.0220 *$ \\
\hline H6 & $0.8731(4)$ & $0.0971(4)$ & $0.5572(4)$ & $0.0199 *$ \\
\hline H8 & $1.2004(4)$ & $0.0320(4)$ & $0.9927(4)$ & $0.0200 *$ \\
\hline H10 & $1.6857(5)$ & $0.7167(4)$ & $1.4851(4)$ & $0.0225^{*}$ \\
\hline H9 & $1.5681(5)$ & $0.4422(4)$ & $1.3781(3)$ & $0.0233^{*}$ \\
\hline
\end{tabular}

Atomic displacement parameters $\left(\AA^{2}\right)$

\begin{tabular}{lllllll}
\hline & $U^{11}$ & $U^{22}$ & $U^{33}$ & $U^{12}$ & $U^{13}$ & $U^{23}$ \\
\hline C1 & $0.0143(15)$ & $0.0208(16)$ & $0.0146(15)$ & $0.0014(12)$ & $0.0026(12)$ & $0.0011(12)$ \\
C2 & $0.0178(17)$ & $0.0183(16)$ & $0.0186(16)$ & $0.0025(13)$ & $0.0082(13)$ & $0.0038(13)$ \\
C3 & $0.0128(16)$ & $0.0187(16)$ & $0.0184(16)$ & $0.0050(12)$ & $0.0089(12)$ & $0.0049(12)$ \\
C4 & $0.0201(17)$ & $0.0165(16)$ & $0.0173(16)$ & $-0.0003(13)$ & $0.0078(13)$ & $0.0011(12)$ \\
O5 & $0.0245(13)$ & $0.0198(12)$ & $0.0198(12)$ & $0.0046(10)$ & $0.0026(10)$ & $0.0052(9)$ \\
O6 & $0.0278(13)$ & $0.0152(12)$ & $0.0220(12)$ & $0.0035(9)$ & $0.0016(10)$ & $0.0025(9)$ \\
O7 & $0.0300(13)$ & $0.0159(11)$ & $0.0199(11)$ & $0.0037(10)$ & $-0.0006(10)$ & $0.0043(9)$ \\
O8 & $0.0236(12)$ & $0.0165(12)$ & $0.0159(11)$ & $0.0014(9)$ & $0.0030(9)$ & $0.0024(9)$ \\
C9 & $0.0158(16)$ & $0.0195(16)$ & $0.0174(15)$ & $0.0004(13)$ & $0.0070(13)$ & $-0.0008(12)$ \\
C10 & $0.0166(16)$ & $0.0177(16)$ & $0.0208(16)$ & $0.0040(12)$ & $0.0071(13)$ & $0.0041(12)$ \\
C11 & $0.0184(16)$ & $0.0215(17)$ & $0.0185(16)$ & $0.0051(13)$ & $0.0088(13)$ & $0.0064(13)$ \\
C12 & $0.0145(16)$ & $0.0202(16)$ & $0.0157(16)$ & $0.0032(12)$ & $0.0076(13)$ & $0.0038(12)$
\end{tabular}




$\begin{array}{lllllll}\text { C13 } & 0.0200(17) & 0.0145(15) & 0.0189(16) & 0.0013(13) & 0.0074(13) & 0.0012(12) \\ \text { C14 } & 0.0172(16) & 0.0237(17) & 0.0153(15) & 0.0029(13) & 0.0042(13) & 0.0007(12) \\ \text { O15 } & 0.0246(13) & 0.0154(11) & 0.0195(12) & 0.0037(9) & 0.0001(10) & -0.0012(9) \\ \text { C16 } & 0.0132(15) & 0.0171(15) & 0.0195(15) & 0.0030(12) & 0.0063(13) & 0.0048(12) \\ \text { C17 } & 0.0154(15) & 0.0212(17) & 0.0172(15) & 0.0065(13) & 0.0075(12) & 0.0064(12) \\ \text { C18 } & 0.0216(17) & 0.0217(16) & 0.0148(15) & 0.0042(13) & 0.0063(13) & 0.0074(12) \\ \text { C19 } & 0.0252(17) & 0.0146(16) & 0.0266(18) & 0.0049(13) & 0.0028(14) & 0.0033(13)\end{array}$

Geometric parameters $\left(\AA,{ }^{\circ}\right)$

\begin{tabular}{|c|c|c|c|}
\hline $\mathrm{C} 1-\mathrm{C} 13$ & $1.392(4)$ & $\mathrm{O} 8-\mathrm{C} 12$ & $1.364(3)$ \\
\hline $\mathrm{C} 1-\mathrm{C} 17$ & $1.369(4)$ & $\mathrm{C} 9-\mathrm{C} 10$ & $1.376(4)$ \\
\hline $\mathrm{C} 1-\mathrm{H} 6$ & 1.000 & $\mathrm{C} 9-\mathrm{C} 14$ & $1.400(5)$ \\
\hline $\mathrm{C} 2-\mathrm{C} 11$ & $1.441(4)$ & $\mathrm{C} 9-\mathrm{O} 15$ & $1.362(4)$ \\
\hline $\mathrm{C} 2-\mathrm{C} 12$ & $1.401(4)$ & $\mathrm{C} 10-\mathrm{H} 4$ & 1.000 \\
\hline $\mathrm{C} 2-\mathrm{C} 17$ & $1.425(4)$ & $\mathrm{C} 12-\mathrm{C} 16$ & $1.376(4)$ \\
\hline $\mathrm{C} 3-\mathrm{C} 4$ & $1.387(4)$ & $\mathrm{C} 13-\mathrm{C} 16$ & $1.390(4)$ \\
\hline $\mathrm{C} 3-\mathrm{C} 10$ & $1.404(4)$ & $\mathrm{C} 14-\mathrm{C} 18$ & $1.372(4)$ \\
\hline $\mathrm{C} 3-\mathrm{C} 11$ & $1.454(4)$ & $\mathrm{C} 14-\mathrm{H} 10$ & 1.000 \\
\hline $\mathrm{C} 4-\mathrm{O} 8$ & $1.373(4)$ & $\mathrm{O} 15-\mathrm{C} 19$ & $1.422(4)$ \\
\hline $\mathrm{C} 4-\mathrm{C} 18$ & $1.401(4)$ & $\mathrm{C} 16-\mathrm{H} 8$ & 1.000 \\
\hline $\mathrm{O} 5-\mathrm{C} 11$ & $1.256(4)$ & $\mathrm{C} 18-\mathrm{H} 9$ & 1.000 \\
\hline $\mathrm{O} 6-\mathrm{C} 13$ & $1.352(4)$ & $\mathrm{C} 19-\mathrm{H} 1$ & 1.000 \\
\hline O6- $\mathrm{H} 5$ & 0.819 & $\mathrm{C} 19-\mathrm{H} 3$ & 1.000 \\
\hline $\mathrm{O} 7-\mathrm{C} 17$ & $1.349(4)$ & $\mathrm{C} 19-\mathrm{H} 2$ & 1.000 \\
\hline $\mathrm{O} 7-\mathrm{H} 7$ & 0.820 & & \\
\hline $\mathrm{C} 13-\mathrm{C} 1-\mathrm{C} 17$ & $119.9(3)$ & $\mathrm{C} 2-\mathrm{C} 12-\mathrm{C} 16$ & $122.8(3)$ \\
\hline $\mathrm{C} 13-\mathrm{C} 1-\mathrm{H} 6$ & 120.0 & $\mathrm{O} 8-\mathrm{C} 12-\mathrm{C} 16$ & $115.7(3)$ \\
\hline $\mathrm{C} 17-\mathrm{C} 1-\mathrm{H} 6$ & 120.0 & $\mathrm{C} 1-\mathrm{C} 13-\mathrm{O} 6$ & $116.8(3)$ \\
\hline $\mathrm{C} 11-\mathrm{C} 2-\mathrm{C} 12$ & $120.8(3)$ & $\mathrm{C} 1-\mathrm{C} 13-\mathrm{C} 16$ & $121.1(3)$ \\
\hline $\mathrm{C} 11-\mathrm{C} 2-\mathrm{C} 17$ & $122.4(3)$ & $\mathrm{O} 6-\mathrm{C} 13-\mathrm{C} 16$ & $122.0(3)$ \\
\hline $\mathrm{C} 12-\mathrm{C} 2-\mathrm{C} 17$ & $116.8(3)$ & $\mathrm{C} 9-\mathrm{C} 14-\mathrm{C} 18$ & $120.2(3)$ \\
\hline $\mathrm{C} 4-\mathrm{C} 3-\mathrm{C} 10$ & $118.7(3)$ & $\mathrm{C} 9-\mathrm{C} 14-\mathrm{H} 10$ & 119.8 \\
\hline $\mathrm{C} 4-\mathrm{C} 3-\mathrm{C} 11$ & $119.7(3)$ & $\mathrm{C} 18-\mathrm{C} 14-\mathrm{H} 10$ & 119.8 \\
\hline $\mathrm{C} 10-\mathrm{C} 3-\mathrm{C} 11$ & $121.6(3)$ & $\mathrm{C} 9-\mathrm{O} 15-\mathrm{C} 19$ & $117.9(2)$ \\
\hline $\mathrm{C} 3-\mathrm{C} 4-\mathrm{O} 8$ & $122.8(3)$ & $\mathrm{C} 13-\mathrm{C} 16-\mathrm{C} 12$ & $118.4(3)$ \\
\hline $\mathrm{C} 3-\mathrm{C} 4-\mathrm{C} 18$ & $121.4(3)$ & $\mathrm{C} 13-\mathrm{C} 16-\mathrm{H} 8$ & 120.8 \\
\hline $\mathrm{O} 8-\mathrm{C} 4-\mathrm{C} 18$ & $115.8(3)$ & $\mathrm{C} 12-\mathrm{C} 16-\mathrm{H} 8$ & 120.8 \\
\hline $\mathrm{C} 13-\mathrm{O} 6-\mathrm{H} 5$ & 109.4 & $\mathrm{C} 2-\mathrm{C} 17-\mathrm{C} 1$ & $121.0(3)$ \\
\hline $\mathrm{C} 17-\mathrm{O} 7-\mathrm{H} 7$ & 109.0 & $\mathrm{C} 2-\mathrm{C} 17-\mathrm{O} 7$ & $120.8(3)$ \\
\hline $\mathrm{C} 4-\mathrm{O} 8-\mathrm{C} 12$ & $119.4(2)$ & $\mathrm{C} 1-\mathrm{C} 17-\mathrm{O} 7$ & $118.2(3)$ \\
\hline $\mathrm{C} 10-\mathrm{C} 9-\mathrm{C} 14$ & $120.6(3)$ & $\mathrm{C} 4-\mathrm{C} 18-\mathrm{C} 14$ & $119.1(3)$ \\
\hline $\mathrm{C} 10-\mathrm{C} 9-\mathrm{O} 15$ & $125.2(3)$ & $\mathrm{C} 4-\mathrm{C} 18-\mathrm{H} 9$ & 120.4 \\
\hline $\mathrm{C} 14-\mathrm{C} 9-\mathrm{O} 15$ & $114.2(3)$ & $\mathrm{C} 14-\mathrm{C} 18-\mathrm{H} 9$ & 120.4 \\
\hline $\mathrm{C} 3-\mathrm{C} 10-\mathrm{C} 9$ & $120.0(3)$ & $\mathrm{O} 15-\mathrm{C} 19-\mathrm{H} 1$ & 109.4 \\
\hline $\mathrm{C} 3-\mathrm{C} 10-\mathrm{H} 4$ & 120.0 & $\mathrm{O} 15-\mathrm{C} 19-\mathrm{H} 3$ & 109.4 \\
\hline $\mathrm{C} 9-\mathrm{C} 10-\mathrm{H} 4$ & 120.0 & $\mathrm{H} 1-\mathrm{C} 19-\mathrm{H} 3$ & 109.4 \\
\hline
\end{tabular}


supporting information

\begin{tabular}{llll}
$\mathrm{C} 3-\mathrm{C} 11-\mathrm{C} 2$ & $115.8(3)$ & $\mathrm{O} 15-\mathrm{C} 19-\mathrm{H} 2$ & 109.4 \\
$\mathrm{C} 3-\mathrm{C} 11-\mathrm{O} 5$ & $122.0(3)$ & $\mathrm{H} 1-\mathrm{C} 19-\mathrm{H} 2$ & 109.4 \\
$\mathrm{C} 2-\mathrm{C} 11-\mathrm{O} 5$ & $122.2(3)$ & $\mathrm{H} 3-\mathrm{C} 19-\mathrm{H} 2$ & 109.4 \\
$\mathrm{C} 2-\mathrm{C} 12-\mathrm{O} 8$ & $121.5(3)$ & & \\
\hline
\end{tabular}

Hydrogen-bond geometry $\left(\AA,{ }^{\circ}\right)$

\begin{tabular}{lllll}
\hline$D-\mathrm{H} \cdots A$ & $D-\mathrm{H}$ & $\mathrm{H} \cdots A$ & $D \cdots A$ & $D-\mathrm{H} \cdots A$ \\
\hline $\mathrm{O} 6-\mathrm{H} 5 \cdots \mathrm{O} 5^{\mathrm{i}}$ & 0.82 & 2.00 & $2.738(3)$ & 150 \\
$\mathrm{O} 7-\mathrm{H} 7 \cdots \mathrm{O} 5$ & 0.82 & 1.91 & $2.634(3)$ & 147 \\
\hline
\end{tabular}

Symmetry code: (i) $x, y-1, z$. 